\title{
Strafrechtliche Behandlungsmassnahmen abseits von zuverlässigen Diagnosen
}

\author{
Chris Lehner *
}

Anmerkung zu den Urteilen des Bundesgerichts 6B_933/2018 vom 3. Oktober 2019 und 6B_828/2019 vom 5. November 2019: Das Bundesgericht verzichtet beim Eingangskriterium der schweren psychischen Störung gemäss Art. 63 und 59 StGB teilweise auf das Erfordernis einer Diagnose nach den international anerkannten Klassifikationssystemen ICD und DSM. Es erweitert damit den Kreis möglicher Massnahmenpatienten in unberechenbarem Ausmass. Die Praxisänderung missachtet die eindeutige Gesetzeslage, stösst in forensisch-psychiatrischen Kreisen auf breiten Widerspruch und stellt die Rechtfertigung therapeutischer Massnahmen infrage.

I. Kritik an der bestehenden Regelung ...................................................... 148

II. Änderung der Rechtsprechung durch das Bundesgericht ............................... 148

1. Urteil des Bundesgerichts 6B_933/2018 vom 3. Oktober 2019 ................... 149

2. Urteil des Bundesgerichts 6B_828/2019 vom 5. November 2019 .................151

III. Anmerkungen zu den zwei Urteilen des Bundesgerichts................................. 153

1. Missachtung von Gesetzeswortlaut und Entstehungsgeschichte der Norm .. 154

2. «Funktionale» Natur des Begriffs der psychischen Störung........................ 156

3. Verzicht auf die Anwendung international anerkannter

Klassifikationssysteme .................................................................. 158

4. Beurteilung des Schweregrads der Störung............................................... 159

5. Tragweite der neuen bundesgerichtlichen Praxis.....................................160

Zitiervorschlag: Chris Lehner, Strafrechtliche Behandlungsmassnahmen abseits von zuverlässigen Diagnosen, in: sui-generis 2020, S. 147

URL: $\quad$ sui-generis.ch/127

DOI: $\quad$ https://doi.org/10.21257/sg.127

* Dr. iur. Chris Lehner (chris.lehner@lu.ch), Richter am Kantonsgericht Luzern. Chris Lehner vertritt in diesem Beitrag seine persönliche Meinung. Dr. iur. Jonas Achermann, Prof. Dr. med. Elmar Habermeyer und MLaw Stefan Wiprächtiger danke ich herzlich für die kritische Durchsicht des Beitrags und ihre wertvollen Hinweise.

Dieses Werk ist lizenziert unter einer Creative Commons Namensnennung - Weitergabe unter gleichen Bedingungen 4.0 International Lizenz. 


\section{Kritik an der bestehenden Regelung}

1 In einem Gastkommentar der Neuen Zürcher Zeitung vom Januar $2018^{1}$ beanstandeten Thomas Noll und Jérôme Endrass die ihrer Ansicht nach zu einschränkende Regelung von Art. 59 StGB: Das Gesetz fordere, dass ein Täter zwingend psychisch krank sein müsse, damit eine stationäre therapeutische Massnahme angeordnet werden könne. Dies führe unter anderem zu grossen Sicherheitsrisiken, denn es gebe «gefährliche und therapierbare Straftäter, die nicht behandelt werden, weil keine offizielle Krankheit vorliegt». Im April 2018 doppelte Frank Urbaniok in der NZZ am Sonntag nach:2 Die Fixierung auf psychiatrische Diagnosen habe fatale Folgen für die Sicherheit der Bevölkerung. Für eine Frau, die vergewaltigt oder für einen Passanten, der spitalreif geschlagen werde, sei es belanglos, ob der Täter in irgendeinem Diagnosesystem als krank oder als gesund gelte. Darum sollten sich nach seiner Ansicht auch Justiz und Psychiatrie vor allem an der Gefährlichkeit und den Risiko-Eigenschaften einer Person orientieren.

\section{II. Änderung der Rechtsprechung durch das Bundesgericht}

2 Was genau unter dem in Art. 63 und 59 StGB verwendeten Begriff der «schweren psychischen Störung» zu verstehen ist, und nach welchen Kriterien sich namentlich der Schweregrad dieser Störung bestimmen lässt, hat das Bundesgericht

\footnotetext{
Gastkommentar in der NZZ vom 31. Januar 2018. «Psychiater Frank Urbaniok fordert, dass Straftäter besser beurteilt werden. Er weiss wie» in der NZZ am Sonntag vom 14. April 2018.
}

bisher nie eindeutig geklärt. 3 Die diesbezügliche höchstrichterliche Rechtsprechung war vielmehr einzelfallbezogen; allgemeingültige Kriterien für die Identifikation einer hinreichend ausgeprägten seelischen Beeinträchtigung des Straftäters 4 fanden sich in den einschlägigen Urteilen aus Lausanne bis anhin keine. 5 Bereits die Frage, ob es sich bei diesem Kriterium um eine Tat- oder Rechtsfrage handelt, beantwortete das Bundesgericht in der Vergangenheit wiederholt uneinheitlich. ${ }^{6}$

3 In zwei Urteilen vom Oktober / November 2019 äusserte sich das Bundesgericht sodann ungewohnt deutlich zum Störungsbegriff nach Art. 63 und 59 StGB. Die einleitend erwähnte, lauthals geäusserte Kritik scheint ihre Wirkung in Lausanne dabei nicht verfehlt zu haben: Das Bundesgericht stellt den zentralen Gehalt des Rechtsbegriffs der schweren psychischen Störung, der in diesen zwei Urteilen erstmals höchstrichterlich in dieser Ausführlichkeit behandelt wurde,

$3 \longdiv { \text { Gunhild Godenzi, Die «schwere psychische Stö- } }$ rung» - grundsätzliche Bemerkungen, in: Marianne Heer/Elmar Habermeyer/Stephan Bernard (Hrsg.), Die schwere psychische Störung als Voraussetzung von therapeutischen Massnahmen, 2019, S. 3 ff., S. 11; Marianne Heer, Kriterien für eine Umschreibung der Schwere einer psychischen Störung gemäss Art. 59 und Art. 63 StGB, in: Marianne Heer/Elmar Habermeyer/Stephan Bernard (Hrsg.), Die schwere psychische Störung als Voraussetzung von therapeutischen Massnahmen, 2019, S. 25 ff., S. 29 f.

4 Zum Zweck der besseren Lesbarkeit wird in diesem Beitrag stets die männliche Form verwendet, mitgemeint sind hier und im Folgenden jedoch stets auch Straftäterinnen.

5 Chris Lehner, Das Kriterium der schweren psychischen Störung - Die Rechtsprechung des Schweizerischen Bundesgerichts, in: Marianne Heer/Elmar Habermeyer/Stephan Bernard (Hrsg.), Die schwere psychische Störung als Voraussetzung von therapeutischen Massnahmen, 2019, S. $85 \mathrm{ff}$, S. $96 \mathrm{ff}$.

6 Lehner (Fn. 5), S. 89 ff. 
zur Überraschung vieler sogleich infrage. Die beiden Entscheide werden nachfolgend zusammenfassend wiedergegeben und im Anschluss kurz kommentiert. Der Schwerpunkt liegt dabei auf dem zur amtlichen Publikation vorgesehenen Entscheid.

\section{Urteil des Bundesgerichts 6B_933/2018 vom 3. Oktober 2019}

\section{a) Sachverhalt}

4 Der Beschuldigte A war der Anführer seiner «Raja-Yoga-Schule», einer Mediationsgruppe mit sektenähnlichen Strukturen. Die Lebensführung seiner «Schüler» bestimmte der selbsternannte «höchsterleuchtete Mann auf Erden» in autoritärer Art und allumfassend. Die aus dem Kreis seiner «Schüler» stammenden späteren Opfer, die sich in schwierigen Lebenssituationen befanden, soll er gemäss Vorhalt der Staatsanwaltschaft systematisch ausgenützt haben. Durch das von ihm geschaffene Klima der Verunsicherung und Angst sei es A möglich gewesen, den Alltag seiner Opfer $\mathrm{zu}$ kontrollieren und $\mathrm{zu}$ lenken sowie namentlich sexuelle Handlungen von ihnen einzufordern. Die Staatsanwaltschaft warf dem Beschuldigten in diesem Zusammenhang unter anderem zahlreiche Verbrechen gegen die sexuelle Integrität vor.7

5 Am 6. Dezember 2016 befand das Bezirksgericht Zurzach A unter anderem der mehrfachen, teilweise qualifizierten sexuellen Nötigung zum Nachteil von drei Frauen für schuldig und bestrafte ihn mit einer Freiheitsstrafe von 9 Jahren

Urteil des Bundesgerichts 6B_933/2018 vom 3. Oktober 2019 (zur Publikation vorgesehen) Sachverhalt lit. A. und 3 Monaten. Gleichzeitig widerrief es eine im Jahr 2009 bedingt ausgesprochene Freiheitsstrafe von 21 Monaten. Das Bezirksgericht ordnete überdies die Verwahrung an. ${ }^{8}$

6 Auf Berufung des Beschuldigten hin bestätigte das Aargauer Obergericht die Schuldsprüche grossmehrheitlich und bestrafte ihn mit einer Freiheitsstrafe von neun Jahren, teilweise als Zusatzstrafe zur im Jahr 2009 ergangenen Verurteilung. Auf die Anordnung der Verwahrung verzichtete es, sprach stattdessen jedoch eine ambulante Massnahme nach Art. 63 StGB aus. 9

7 Die gegen das obergerichtliche Urteil geführte Beschwerde in Strafsachen, mit der sich der Beschuldigte insbesondere gegen die Anordnung der ambulanten Massnahme wehrte, wies das Bundesgericht mit Urteil vom 3. Oktober 2019 ab.

\section{b) Erwägungen}

8 Hinsichtlich der angeordneten ambulanten Massnahme führte das Obergericht des Kantons Aargau im angefochtenen Entscheid aus, nachgewiesen seien bei A aus medizinischer Sicht zumindest Merkmale einer akzentuierten narzisstischen Persönlichkeit. Aufgrund ihrer hohen Ausprägung in den vergangenen zehn bis fünfzehn Jahren seien diese als «Persönlichkeitsmerkmal mit Störcharakter» zu bezeichnen. Der Gutachter habe zusätzlich einen sehr ausgeprägten «Dominanzfokus» (mit sehr hoher Bedeutung für das Tatverhalten) festgestellt. Insgesamt liege zwar keine psychi-

8 Urteil des Bundesgerichts 6B_933/2018 vom 3. Oktober 2019 Sachverhalt lit. B.

9 Urteil des Bundesgerichts 6B_933/2018 vom 3. Oktober 2019 Sachverhalt lit. $\bar{C}$. 
sche Störung im engeren Sinne vor, jedoch seien langanhaltende deliktrelevante Persönlichkeitsmerkmale mit Krankheitswert gegeben. Die medizinischen Feststellungen reichten aus, so die Vorinstanz, um eine schwere psychische Störung im massnahmenrechtlichen Sinne anzunehmen. ${ }^{10}$

9 Das Bundesgericht befasst sich ab Erwägung 3.5 mit dem Begriff der schweren psychischen Störung nach Art. 63 StGB. In diesem Zusammenhang wiederholt es die bisher in diesem Bereich ergangene, wie erwähnt nicht besonders ergiebige höchstrichterliche Rechtsprechung. ${ }^{11}$ Ergänzend dazu führt es aus, der Rechtsbegriff der schweren psychischen Störung entspreche einem medizinischen Substrat, einem Defizit mit Krankheitswert. Der Begriff sei funktionaler Natur, da er sich nach dem Zweck der Massnahme, der Verminderung der Rückfallgefahr, richte. Eine Verbesserung des Gesundheitszustands des Täters interessiere das Strafrecht allenfalls nur mittelbar - insoweit sie der Deliktsprävention diene. ${ }^{12}$

Immerhin stellt das Bundesgericht im Anschluss daran fest, sei der funktionale Gesichtspunkt nicht allein begriffsbildend. Es verweist dabei namentlich auf dissoziale Straftäter und hält fest, dass die Übergänge zwischen einem bloss kriminellen Lebensstil und einer dissozialen Persönlichkeitsstörung mit Krankheitswert fliessend sein könnten und es zu verhindern gelte, gewöhnliche Kriminalität zu pathologisieren. Insbesondere

10 Urteil des Bundesgerichts 6B_933/2018 vom 3. Oktober 2019 E. 3.5.1.

11 Urteil des Bundesgerichts 6B_933/2018 vom 3. Oktober 2019 E. 3.5.2.

12 Zum Ganzen: Urteil des Bundesgerichts 6B_933/2018 vom 3. Oktober 2019 E. 3.5.3. bei (dissozialen) Persönlichkeitsstörungen sei deshalb auf eine rein kategoriale Unterscheidung nach Diagnosen und Schweregraden zu verzichten. Das Bundesgericht hält weiter dafür, dass fest mit der Persönlichkeit verbundene Merkmale grundsätzlich eine psychische Störung im Rechtssinne indizierten. Massgebend sei, ob sich die im Zusammenhang mit der Tat stehenden Persönlichkeitsmerkmale auch in nichtdeliktischen Lebensbereichen dysfunktional auswirkten, ob das psychosoziale Funktionsniveau mithin insgesamt stark eingeschränkt sei. Die letztgenannten Kriterien kämen indes nicht immer zum Tragen, zu denken sei etwa an Störungen, die sich wegen ihrer hohen Spezifität nur im kriminellen Handeln ausdrückten, während das beobachtbare Verhalten eher unauffällig bleibe. ${ }^{13}$

11 In der Folge gelangt das Bundesgericht zur Kernaussage des Leitentscheids, indem es festhält, dass in Fällen, in denen die gutachterliche Diagnose nicht nach den gängigen Klassifikationssystemen (ICD oder DSM) eingeordnet werden könne, eine gesicherte Feststellung einer schweren psychischen Störung im Rechtssinn gleichwohl möglich sei. Voraussetzung dafür sei, dass die Diagnose auf delikt- und risikorelevanten persönlichkeitsnahen Risikofaktoren beruhe, die einer risikovermindernden Therapie zugänglich seien. Es begründet dies damit, dass die genannten Diagnosemanuale in der forensischen Psychiatrie für einen Zweck verwendet würden, für den sie nicht geschaffen worden seien. Diese dienten in erster Linie einer Vereinheitlichung des internationalen Umgangs mit

13 Zum Ganzen: Urteil des Bundesgerichts 6B_933/2018 vom 3. Oktober 2019 E. 3·5.4. 
Diagnosen. Ein gemäss ICD-10 oder DSM-V kodierter Befund erfülle indes (aufgrund der grossen Bandbreite des dort definierten Störungsbegriffs) nicht ohne Weiteres den gesetzlichen Begriff einer schweren psychischen Störung. Strafrechtliche Behandlungen seien deliktorientiert und unterschieden sich deshalb von der allgemeinmedizinischen Versorgung, auf die die Klassifikationssysteme abzielten. ${ }^{14}$

Die Bestimmung des Schweregrads der «psychischen Störung» im Rechtssinne will das Bundesgericht schliesslich daran festmachen, mit welcher Intensität sich die Störung in der Tat manifestiert. Eine bestimmte Diagnose könne deshalb nicht per se als schwer im Sinne des Gesetzes bezeichnet werden, vielmehr könne auch eine Kombination von für sich genommen wenig ausgeprägten Befunden im Ergebnis eine schwere psychische Störung ergeben. ${ }^{15}$

Auf den konkreten Fall angewendet folgert das Bundesgericht, dass der Beschuldigte im Sinne von Art. 63 StGB psychisch schwer gestört sei. Der beigezogene Sachverständige habe eine moderat auffällige akzentuierte narzisstische Persönlichkeit (Z73.1 nach ICD-10) sowie einen sehr stark ausgeprägten sogenannten «Dominanzfokus», bestehend aus Kontrollbedürfnis, Dominanzstreben und Ignorieren der Bedürfnisse anderer festgestellt. Gemäss Gutachten handle es sich beim erstgenannten Befund um «deliktrelevante Persönlichkeitsmerkmale mit Krankheitswert». Der Sachverständi-

14 Zum Ganzen: Urteil des Bundesgerichts 6B_933/2018 vom 3. Oktober 2019 E. 3.5.5.

15 Zum Ganzen: Urteil des Bundesgerichts 6B_933/2018 vom 3. Oktober 2019 E. 3.5.6. ge war sich offenbar nicht sicher, ob sich der «Dominanzfokus» auch in nichtdeliktischen Lebensbereichen dysfunktional auswirkt, führte er doch aus, es sei auch möglich, dass sich $\mathrm{A}$ in anderen Bereichen nicht dominant verhalte. Aufgrund der Tatsache, dass gemäss Gutachter persönlichkeitsnahe und nicht situative Faktoren zur Delinquenz geführt hätten, die Taten genauer durch die manipulativen Fähigkeiten des Täters ermöglicht worden seien, hegt das Bundesgericht indes keine Zweifel am Vorliegen einer schweren psychischen Störung im Rechtssinne. Den hinreichenden Schweregrad der Störung begründet nach Ansicht des Bundesgerichts sodann der Umstand, dass dem «Dominanzfokus» sehr grosse Bedeutung für das Tatverhalten zukomme. Gemäss Gutachter liessen die seit Jahren bestehende sektenähnliche Struktur der Meditationsgruppe und das Verhalten von A gegenüber den betroffenen Frauen den Schluss auf ein stark ausgeprägtes Dominanz- und Kontrollbedürfnis $\mathrm{zu}$, das auch für die begangenen Delikte sehr relevant sei. Demgegenüber erkläre die akzentuierte Persönlichkeit des Beschuldigten allein das Tatverhalten nicht, ihr komme maximal moderate Bedeutung für die Tatentstehung zu. ${ }^{16}$

\section{Urteil des Bundesgerichts 6B_828/2019 vom 5. November 2019}

\section{a) Sachverhalt}

14 A wurde mit Urteil des Strafgerichts Basel-Landschaft vom 21. August 2014 der Vergewaltigung, der versuchten Verge-

16 Zum Ganzen: Urteil des Bundesgerichts 6B_933/2018 vom 3. Oktober 2019 E. 3.5.7. 
waltigung, der versuchten sexuellen Handlungen mit einem Kind, der Pornografie und der Widerhandlung gegen das Waffengesetz schuldig gesprochen und mit einer Freiheitsstrafe von 7 Jahren bestraft. Für ihn wurde unter Aufschub der Freiheitsstrafe eine Massnahme für junge Erwachsene nach Art. 61 StGB angeordnet. Das Urteil des Strafgerichts erwuchs unangefochten in Rechtskraft. ${ }^{17}$

15 Nachdem die Vollzugsbehörde die Massnahme nach Art. 61 StGB infolge Erreichens der gesetzlichen Höchstdauer aufgehoben hatte, ordnete der Präsident des Strafgerichts Basel-Landschaft am 23. August 2018 auf Gesuch der Vollzugsbehörde für A eine Massnahme nach Art. 59 StGB an. Die dagegen erhobene Beschwerde wies das Kantonsgericht Basel-Landschaft mit Beschluss vom 22. Januar 2019 ab. ${ }^{18}$

16 A gelangte daraufhin ans Bundesgericht und beantragte unter anderem die Aufhebung der stationären therapeutischen Massnahme. Das Bundesgericht wies die Beschwerde mit Urteil vom 5. November 2019 ab.

\section{b) Erwägungen}

Das Bundesgericht bestätigt in diesem Urteil seine rund einen Monat zuvor im Urteil 6B_933/2018 vom 3. Oktober 2019 zum Begriff der schweren psychischen Störung nach Art. 63 StGB entwickelte Rechtsprechung erstmals und überträgt sie auf die stationäre therapeutische Massnahme nach Art. 59 StGB.

17 Urteil des Bundesgerichts 6B_828/2019 vom 5. November 2019 Sachverhalt lit. A.

18 Urteil des Bundesgerichts 6B_828/2019 vom 5. November 2019 Sachverhalt lit. B und C.
18 Zunächst beschäftigt es sich mit dem Gutachten des beigezogenen forensischen Psychiaters. Dieser stellte bei A neben akzentuierten dissozialen Persönlichkeitszügen und einem «Zustand nach einer einfachen Aktivitäts- und Aufmerksamkeitsstörung» eine «Vergewaltigungsdisposition» fest. Letztere beschrieb der Sachverständige als Wünsche und Handlungsimpulse des Täters, sexuelle Handlungen gegen oder unabhängig vom Willen der geschädigten Person zu erzwingen. Gewaltsam herbeigeführte Sexualkontakte würden von den Betroffenen als attraktiv erlebt oder als legitime Strategie der eigenen Bedürfnisbefriedigung angesehen und mit entsprechenden Fantasien einhergehen. Die «Vergewaltigungsdisposition» erfasste der Gutachter in der Restkategorie der sonstigen Störungen der Sexualpräferenz (F65.8 nach ICD-10), da sie in der ICD-10 nicht explizit aufgeführt sei. In Kombination mit den akzentuierten dissozialen Persönlichkeitszügen müsse bei A eine schwere psychische Störung diagnostiziert werden. ${ }^{19}$

19 Das Bundesgericht bestätigt in der Folge die Vorinstanz, soweit sie die "gutachterliche Diagnose der Vergewaltigungsdisposition» in Kombination mit den dissozialen Persönlichkeitszügen als psychische Störung im Sinne von Art. 59 Abs. 1 StGB qualifiziert. Begründend hält es fest, der Gutachter lege dar, dass die Vergewaltigungsdisposition in der forensischen Psychiatrie anerkannt sei, auch wenn dafür andere Begriffe wie Vergewaltigungsfantasien oder Vergewaltigungsneigung verwendet würden. Die Rechtsprechung habe kürzlich (im Urteil 6B_933/2018

19 Urteil des Bundesgerichts 6B_828/2019 vom 5. November 2019 E. 1.3. 
vom 3. Oktober 2019) bestätigt, dass die Diagnose nicht unter allen Umständen in einem Identifikationssystem wie ICD oder DSM aufgeführt sein müsse. Dass diese Manuale den Begriff der Vergewaltigungsdisposition nicht kennen, spreche daher nicht gegen die Annahme einer schweren psychischen Störung im Rechtssinne. Der Gutachter lege ausführlich dar, weshalb er beim Verurteilten von einer Vergewaltigungsdisposition, d.h. dem Wunsch nach sexuellen Handlungen gegen den Willen der betroffenen Person, ausgehe. Diese sei nicht deshalb diagnostiziert worden, weil er eine Vergewaltigung begangen habe, sondern weil er nach Auffassung des Gutachters gewaltsam herbeigeführte Sexualkontakte als besonders attraktiv erlebe. ${ }^{20}$

\section{Anmerkungen zu den zwei Urteilen des Bundesgerichts}

Die Lektüre der zwei dargestellten Bundesgerichtsentscheide lässt den unbefangenen Leser auf Anhieb kaum vermuten, dass das Massnahmenrecht damit weitreichende Veränderungen erfahren hat. Namentlich scheinen zahlreiche in den Erwägungen enthaltene Belegstellen aus Rechtsprechung und Literatur längst vorzugeben, was das Bundesgericht nunmehr explizit festhält. ${ }^{21}$ Verschwiegen wird indes, dass das Vorliegen einer nach den gängigen Klassifikationssystemen diagnostizierten psychischen Störung bisher als Selbstverständlichkeit des Massnahmenrechts galt, jedenfalls so-

$20 \overline{\text { Urteil des Bundesgerichts 6B_828/2019 vom }}$ 5. November 2019 E. 1.4.

21 Welcher Eindruck teilweise auch eindeutigen Falschzitaten geschuldet ist, vgl. etwa das bei Felix Bommer, Schwere psychische Störung und schwere systemische Folgen, in: recht 2020, S. 24 ff. auf S. 25 in Fn. 5 aufgedeckte Beispiel. weit es sich um strafrechtliche Behandlungsmassnahmen nach den Art. 59, 60 und 63 StGB handelte. ${ }^{22}$ Die gegenteilige, vom Bundesgericht nun aufgenommene Meinung vertrat in der Schweiz einzig ein enger Kreis von Personen.23 Der Grossteil von ihnen hat das «risikoorientierte Diagnosesystem» FOTRES entweder geschaffen, war an dessen Entwicklung beteiligt oder vertritt dieses nach aussen. Das Instrument wird als (einzig richtige) Alternative $\mathrm{zu}$ den international gebräuchlichen Diagnosemanualen angepriesen. ${ }^{24}$ Daraus stammt auch der vom

22 Siehe nur Bernd Borchard/Juliane Gerth, Alternative zur schweren psychischen Störung nach ICD oder DSM als Voraussetzung für die Anordnung therapeutischer Massnahmen bei Straftätern, in: Marianne Heer/Elmar Habermeyer/ Stephan Bernard (Hrsg.), Die schwere psychische Störung als Voraussetzung von therapeutischen Massnahmen, 2019, S. 59 ff., S. 60; Godenzi (Fn. 3), S. 9; Marianne Heer/Elmar Habermeyer, in: Marcel Alexander Niggli/Hans Wiprächtiger (Hrsg.), Basler Kommentar Strafrecht I, Art. 1110 StGB, Jugendstrafgesetz, 4. Aufl. 2019, N 9 ff. zu Art. 59 StGB; Nicolas Queloz/Rémy Munyankindi, in: Robert Roth/Laurent Moreillon (Hrsg.), Commentaire Romand, Code pénal I, Art. 1-110 CP, 2009, N 6 zu Art. 59 StGB; Günter Stratenwerth, Schweizerisches Strafrecht, Allgemeiner Teil II: Strafen und Massnahmen, 2. Aufl. 2006, N 10 zu § 9; Stefan Trechsel/Barbara Pauen Borer, in: Stefan Trechsel/Marc Pieth, Schweizerisches Strafgesetzbuch, Praxiskommentar, 3. Aufl. 2017, N 4 zu Art. 59 StGB.

23 Siehe etwa Philipp Maier/Frank Urbaniok, Die Anordnung und praktische Durchführung von Freiheitsstrafen und Massnahmen, mit Behandlungskonzepten für erwachsene Straftäter nach Schweizerischem Strafgesetzbuch, 1998, S. 55 f. oder Frank Urbaniok/Jérôme Endrass/Thomas Noll/Astrid Rossegger, Die «psychische Störung» im Massnahmenrecht aus forensisch-psychiatrischer Sicht, in: AJP 2016, S. 1671 ff.; 1677 f.

24 So Frank Urbaniok in seinem oben in Fn. 2 zitierten Gastbeitrag in der NZZ am Sonntag vom 14. April 2018; zuletzt etwa: Bernd Borchard/ Juliane Gerth, Die Relevanz eines kohärenten forensischen Beurteilungs- und Behandlungsprozesses, in: Forens Psychiatr Psychol Kriminol (2020). Es ist bemerkenswert, dass auch die in den beiden hier kommentierten Strafverfahren als Grundlage dienenden Gutachten von einem 
Bundesgericht übernommene Begriff der «risikorelevanten Persönlichkeitsmerkmale» bzw. «Risiko-Eigenschaften».

Umso mehr lohnt sich ein genauer Blick auf die Argumentationslinie, mit der das Bundesgericht diese Kursänderung begründet. Im Anschluss daran ist auf die Tragweite dieser überraschenden und gleichzeitig folgenschweren Neuauslegung des Gesetzesbegriffs der schweren psychischen Störung einzugehen.

\section{Missachtung von Gesetzeswortlaut und Entstehungsgeschichte der Norm}

22 Der Wortlaut des Gesetzes spielt nach gefestigter bundesgerichtlicher Rechtsprechung stets Ausgangspunkt jeder Gesetzesauslegung. ${ }^{25}$ Von ihm darf, soweit er eindeutig ist, nur aus «triftigen Gründen» abgewichen werden, die die Annahme nahelegen, der Gesetzestext ziele am «wahren Sinn» vorbei. ${ }^{26}$ Der tatsächliche Gehalt einer Rechtsnorm muss (und darf) mit anderen Worten nur dann ermittelt werden, wenn der Wortlaut unklar ist, mithin unterschiedliche Deutungen zulässt. ${ }^{27}$

Die Bindung der Gerichte an den Gesetzestext ist Grundbedingung eines demokratischen, auf die Gewaltenteilung bedachten Rechtsstaats und im Bereich des Strafrechts von zentraler Bedeutung. ${ }^{28}$ Die strafrechtliche Abteilung des Bun-

forensischen Psychiater aus diesem Umfeld stammen.

25 Statt vieler: BGE 142 V 402 E. 4.1.

26 BGE 143 I 272 E. 2.2.3.

BGE 143 I 272 E. 2.2.3.

28 Art. 1 StGB; Peter Popp/Anne Berkemeier, in: Marcel Alexander Niggli/Hans Wiprächtiger (Hrsg.), Basler Kommentar Strafrecht I, Art. 1110 StGB, Jugendstrafgesetz, 4. Aufl. 2019, N 4 zu Art. 1 StGB desgerichts scheint sich in den erwähnten Urteilen davon jedoch nicht besonders beeindruckt zu zeigen. Obwohl das Strafgesetzbuch in Art. 59 und 63 StGB mit aller Deutlichkeit festhält, dass für eine therapeutische Massnahme nur Täter in Betracht kommen, die "psychisch schwer gestört» sind, setzt sich das höchste schweizerische Gericht über den Gehalt dieses bis anhin einmütig verstandenen Begriffs hinweg. ${ }^{29}$ Aus psychiatrischer Sicht ist die psychische Störung klar definiert. Im ersterwähnten Urteil wird denn auch nicht verschwiegen, dass dieser Begriff, soweit er in Art. 59 und 63 StGB verwendet wird, dem Diagnosemanual ICD-10 entnommen ist, $3^{\circ}$ mithin dem dort verwendeten medizinischen Terminus entspricht. Es ist bisher von juristischer Seite nicht einmal der Versuch unternommen worden, den Störungsbegriff weiter als im medizinischen Sinne zu fassen, ohne dabei andere Begrifflichkeiten (wie etwa «Persönlichkeitsmerkmale») zu verwenden. Hätten auch für die Anordnung einer Massnahme nach Art. 63 und 59 StGB seelische Zustände reichen sollen, die nicht als Störung gemäss einem anerkannten Klassifikationssystem erfassbar sind, wäre es ein Leichtes gewesen, dies im Gesetz zu verdeutlichen. Die Bestimmung über die Verwahrung macht es vor: Neben der psychischen Störung von erheblicher Schwere nach Art. 64 Abs. 1 lit. b StGB nennt sie in Abs. 1 lit. a namentlich die

$29 \overline{\text { Dass der Begriff der psychischen Störung nicht }}$ anders als im medizinischen Sinne verstanden werden kann, zeigt nicht zuletzt die einleitend angeführte harsche Kritik an der bis dahin geltenden Gesetzeslage.

30 Vgl. Urteil des Bundesgerichts 6B_933/2018 vom 3. Oktober 2019 E. 3.5.5; Botschaft vom 21. September 1998 zur Änderung des Schweizerischen Strafgesetzbuches, BBl 1999 II 1979, S. 2076, Fn. 224. 
Persönlichkeitsmerkmale des Täters als alternative Eingangskriterien dieser Massnahme. Fakt ist jedoch, dass der Gesetzgeber von einer solchen Öffnung der therapeutischen Massnahmen abgesehen, ja sich vielmehr explizit gegen eine entsprechende Ausweitung strafrechtlicher Behandlungen ausgesprochen hat. 31

24 Die Entkopplung des gesetzlichen vom medizinischen Störungsbegriff widerspricht darüber hinaus der aus der Botschaft zum heutigen Art. 59 StGB unzweideutig ersichtlichen Intention des Gesetzgebers. Die Gesetzesmaterialien machen klar, dass die Etablierung des Begriffs der schweren psychischen Störung als Voraussetzung der therapeutischen Massnahmen bereits Resultat eines Entgegenkommens gegenüber jenen Kritikern darstellte, die der im Vorentwurf enthaltenen Regelung als zu wenig weitgehend entgegentraten. ${ }^{32}$ Dieser hatte noch vorgesehen, dass nur für «geistig kranke bzw. geistig schwer behinderte Personen», nicht aber für persönlichkeitsgestörte Straftäter, eine stationäre therapeutische Massnahme angeordnet werden könne. 33 Die letztgenannte Tätergruppe wollte man mit dem nun geltenden Gesetzestext ebenfalls erfassen, um sie nicht dem regulären Strafvollzug zu überlassen, wo eine adäquate Behandlung nicht möglich sei.34 Da der (in der Botschaft im medizinischen Sinne ver-

31 Siehe Bommer (Fn. 21), S. 27; ferner Bericht der Arbeitsgruppe «Verwahrung» zur Änderung des Schweizerischen Strafgesetzbuches in der Fassung vom 13. Dezember 2002, 2004, S. $37 \mathrm{f}$.

32 Botschaft, BBl 1999 II 1979, S. 2076.

33 Bericht zur Revision des Allgemeinen Teils und des Dritten Buches des Strafgesetzbuches und zu einem Bundesgesetz über die Jugendstrafrechtspflege, 1993, S. 79.

34 Botschaft, BBl 1999 II 1979, S. 2076. wendete)35 Begriff der psychischen Störung sehr weit reicht, schränkte man ihn mit dem Zusatz «schwer» ein. Es handelt sich beim Kriterium der psychischen Störung demnach nicht um eine besonders einschneidende Referenzgrösse, sondern um den kleinsten gemeinsamen Nenner für eine wissenschaftliche und verlässliche Umschreibung psychischer Defektzustände. Soweit das Bundesgericht mit Verweis auf dieselbe Stelle in der Botschaft ${ }^{36}$ demnach belegen will, dass geistige Zustände jenseits der psychischen Störung vom Gesetz mitumfasst sein müssen, ignoriert es schlicht die Entstehungsgeschichte der massgebenden Norm sowie die nachfolgenden Entwicklungen im Rahmen der Umsetzung der Volksinitiative über die lebenslängliche Verwahrung, im Zuge welcher auf eine solche Extension von Art. 59 und 63 StGB verzichtet wurde. Zwar trifft die höchstrichterliche Feststellung $37 \mathrm{zu}$, dass der in den gängigen Klassifikationssystemen verwendete Begriff der psychischen Störung mit dem entsprechenden Eingangskriterium für die therapeutischen Massnah-

35 Dass in der Botschaft davon ausgegangen wird, der gesetzliche Begriff der psychischen Störung entspreche jenem der gebräuchlichen Diagnosemanuale, zeigt insbesondere das dort aufgeführte Beispiel von spielsüchtigen Straftätern. So wird darauf hingewiesen, dass auch diese Abhängigkeit - als «pathologisches Glücksspiel» unter den Persönlichkeits- und Verhaltensstörungen bei den ICD-10-Leitlinien eingereiht - eine psychische Störung darstelle und somit unter diese Bestimmung fallen könne; Botschaft, BBl 1999 II 1979, S. 2076.

36 «Es soll <grundsätzlich die ganze Bandbreite der nach wissenschaftlichen Kriterien diagnostizierbaren, vom sog. Normalen abweichenden psychischen Phänomene zu einer therapeutischen Massnahme führen> können»; Urteil des Bundesgerichts 6B_933/2018 vom 3. Oktober 2019 E. 3.5.5 mit Verweis auf die Botschaft, BBl 1999 II 1979, S. 2076.

37 Urteil des Bundesgerichts 6B_933/2018 vom 3. Oktober 2019 E. 3.5.5. 
men nicht deckungsgleich ist. Dies liegt aber daran, dass dieser aufgrund seiner grossen Bandbreite einer präzisierenden Einschränkung (über die zusätzliche Voraussetzung eines hinreichenden Schweregrads) bedarf - also nicht zu eng ist, sondern deutlich zu weit reicht.

Es lässt sich demnach nicht leugnen, dass das Bundesgericht mit seiner eigenwilligen Neufassung des Rechtsbegriffs der schweren psychischen Störung nichts anderes tat, als sich über die eindeutigen Vorgaben des Gesetzgebers hinwegzusetzen. Ein Anlass dazu bestand nicht; weder war der Wortlaut unklar, noch legen die Gesetzesmaterialien oder - wie noch zu zeigen sein wird - Erkenntnisse aus der forensischen Psychiatrie nahe, dass der Wortlaut von Art. 59 und Art. 63 StGB nicht dem wahren Sinn der Bestimmung entsprach. Eine solche Missachtung legislativer Vorgaben ist nicht nur rechtsstaatlich bedenklich, sondern in ihrem Ausmass im sensiblen Bereich freiheitsentziehender Massnahmen nicht zuletzt mit Blick auf die EMRK heikel. Art. 5 Abs. 1 Satz 2 EMRK, der vorsieht, dass die Freiheit «nur auf die gesetzlich vorgeschriebene Weise» entzogen werden darf, schützt neben unzureichend präzisen Gesetzesnormen im Bereich strafrechtlicher Sanktionen 38 auch vor einem bewussten Abweichen von innerstaatlichen Gesetzesvorschriften. 39 Genau Letzteres propagieren jedoch die zwei kommentierten Bundesgerichtsurteile. Dass der Europäische Ge-

${ }_{38}$ Vgl. hierzu im vorliegenden Zusammenhang: Bommer (Fn. 21), S. 28 f.

39 Siehe etwa Urteile des EGMR 89/1997/873/1085 vom 2. September 1998 (Erkalo gegen die Niederlande), N 52-60, und 50213/99 vom 22. Juni 2004 (Tám gegen die Slowakei) sowie Urteil des EGMR 12889/87 vom 10. Juli 1989 (Smiet gegen die Niederlande). richtshof für Menschenrechte darauf bedacht ist, dem Legalitätsprinzip im $\mathrm{Zu}$ sammenhang mit dem Freiheitsentzug bei Straftätern Nachachtung zu verschaffen, zeigte sich jüngst am Beispiel der vollzugsrechtlichen Sicherheitshaft. Weil für die Anordnung von Haft für verurteilte Straftäter zwischen Aufhebung einer Massnahme und allfälliger Anordnung einer Folgemassnahme (bei fehlender Reststrafe) keine explizite gesetzliche Grundlage in der Strafprozessordnung besteht, wandte das Bundesgericht seit dem Jahr 2011 die Art. 221 und $229 \mathrm{ff}$. StPO auf solche Fälle analog an. 40 Der EGMR taxierte dieses Vorgehen in einem konkreten Fall als konventionswidrig. ${ }^{41}$ Angesichts der Schwere des Eingriffs in die Freiheitsrechte des Betroffenen 42 könne die analoge Anwendung der Art. 221 und 229 ff. StPO die fehlende gesetzliche Grundlage für die vollzugsrechtliche Sicherheitshaft nicht ersetzen. 43

\section{2. «Funktionale» Natur des Begriffs der psychischen Störung}

26 Das Bundesgericht bezeichnet den Gesetzesbegriff der psychischen Störung in beiden Entscheiden mehrfach als einen solchen funktionaler Natur. Es verweist dabei auf den Zweck strafrechtlicher Behandlungsmassnahmen - der Verminderung der Rückfallgefahr des Straftäters und will zum Ausdruck bringen, dass sich auch die massnahmenrechtliche Diagno-

$4 0 \longdiv { \text { BGE } 1 4 2 \text { IV } 1 0 5 \text { E. 5.5; Urteil des Bundesgerichts } }$ 1B_24/2020 vom 3. Februar 2020 E. 3.3.

41 Urteil des EGMR 72939/16 vom 3. Dezember 2019 (I.L. gegen die Schweiz) N 43-58.

42 Konkret ging es um 3 Monate und 10 Tage Sicherheitshaft; Urteil des EGMR 72939/16 vom 3. Dezember 2019 (I.L. gegen die Schweiz) N 3.

43 Urteil des EGMR 72939/16 vom 3. Dezember 2019 (I.L. gegen die Schweiz) N 55. 
se danach auszurichten hat. 44 Es bereitet damit das Feld für seine weitergehende Auffassung, dass sich eine den Art. 63 und 59 StGB genügende Diagnose auch in der blossen Feststellung delikt- und risikorelevanter (persönlichkeitsnaher) Risikofaktoren erschöpfen könne. 45

Dass das Ziel jeder therapeutischen Massnahme in der Verbesserung der Legalprognose des Täters liegt, ist unbestritten. Soweit das Bundesgericht die Diagnose einer psychischen Störung indes vorwiegend am Mass der Rückfallwahrscheinlichkeit festmachen will, greift seine Argumentation zu kurz. Eine nach wissenschaftlichen Kriterien gestellte Diagnose beurteilt sich grundsätzlich unabhängig von der Rückfallgefahr des Straftäters. Auch psychisch gesunde Personen können schwerwiegende Straftaten begehen, wie auf der anderen Seite nicht jede geistig beeinträchtigte Person einen besonderen Hang zur Begehung von Delikten aufweist. ${ }^{46}$ Ob der Straftäter neben seiner geistigen Anomalie eine besonders ungünstige Legalprognose aufweist, ist demnach gesondert zu prüfen und bildet entsprechend Gegenstand einer eigenständigen Anordnungsvoraussetzung der Massnahme. Wohin die vorwiegende Orientierung auch der Diagnose an der zu erwartenden Rückfälligkeit führt, beschreibt das Bundesgericht gleicht selbst:47 zur Pathologisierung jeder Art von Kriminalität. Dass es zur Lösung dieses Problems vorschlägt, namentlich bei Persönlichkeitsstörungen

44 Urteil des Bundesgerichts 6B_933/2018 vom 3. Oktober 2019 E. 3.5.3.

45 Urteil des Bundesgerichts 6B_933/2018 vom 3. Oktober 2019 E. 3.5.5.

46 BSK-StGB Heer/Habermeyer (Fn. 22), N 6 f. zu Art. 59 StGB.

47 Urteil des Bundesgerichts 6B_933/2018 vom 3. Oktober 2019 E. 3.5.4. auf eine «rein kategoriale Unterscheidung - nach Diagnosen und Schweregraden» zu verzichten, entbehrt daher nicht einer gewissen Ironie. Um das oft genannte Beispiel der Dissozialität aufzugreifen: Gerade wo Personen allein aufgrund ihrer intensiven deliktischen Lebensweise risikorelevante Persönlichkeitseigenschaften zugeschrieben werden, tut es Not, ihren psychischen Zustand nach zuverlässigen und nachvollziehbaren Kriterien einzuordnen. Wer dies unterlässt, läuft Gefahr, allein aufgrund einer mehrfachen Deliktsbegehung den zirkulären Schluss auf eine psychische Störung zu ziehen, 48 zeichnen sich dissoziale Persönlichkeitsmerkmale doch massgeblich dadurch aus, dass der Betroffene Mühe hat, sich an soziale Normen zu halten, mithin Gesetzesverstösse begeht. Gerade bei dieser Personengruppe soll gemäss dem erstkommentierten Entscheid jedoch auf die Anwendung solcher Kriterien verzichtet werden.

28 Das Bundesgericht sieht in fest mit der Persönlichkeit des Täters verbundenen Merkmalen grundsätzlich das Vorliegen einer psychischen Störung.49 Die Abgrenzung zu blossem sozial abweichendem Verhalten will es danach vornehmen, ob sich diese Persönlichkeitsmerkmale nicht nur in der Delinquenz, sondern auch in anderen Lebensbereichen dysfunktional auswirken, sodass der Täter in seiner sozialen Kompetenz in Lebensführung und -gestaltung insgesamt stark einge-

48 Elmar Habermeyer/Steffen Lau/Henning Hachtel/Marc Graf, Kritische Anmerkungen aus psychiatrisch-psychotherapeutischer Sicht zu den Bundesgerichtsurteilen 6B_933/2018 vom 3. Oktober 2019 und 6B_828/2019 vom 5. November 2019, in: recht 2020, S. 32 ff., S. 34.

49 Urteil des Bundesgerichts 6B_933/2018 vom 3. Oktober 2019 E. 3.5.4. 
schränkt ist.5o Das genannte Merkmal wird in der Literatur als möglicher Gradmesser diskutiert, um schwere von weniger schwerwiegenden psychischen Störungen im medizinischen Sinne zu unterscheiden.51 Als Diagnoseinstrument taugt es freilich nicht, da es keine verlässliche Einordnung des psychischen Zustands erlaubt, sondern nur dessen Ausmass beschreibt. Das Bundesgericht scheint das Kriterium denn auch selbst nicht besonders ernst zu nehmen, wie sich anschaulich im ersten der zwei dargestellten Fälle zeigt. Ob der beim Täter ausgemachte «Dominanzfokus»52 über die von ihm verübten Delikte hinaus von Bedeutung ist, konnte der Sachverständige gerade nicht schlüssig beantworten, jedenfalls ergibt sich solches nicht aus dem Urteil des Bundesgerichts.53 Gleichwohl bejaht dieses ohne Weiteres das Vorliegen einer schweren psychischen Störung im Sinne von Art. 63 StGB.54
50 Urteil des Bundesgerichts 6B_933/2018 vom 3. Oktober 2019 E. 3.5.4.

51 Winfried Rasch, Forensische Psychiatrie, 1986, S. 23; Henning Sass, Die psychopathologische Konzeption der Schuldfähigkeitsbegutachtung, in: Franz Riklin (Hrsg.), Kapituliert die Strafjustiz vor der Psychiatrie?, 2016, S. 33 ff., S. 36 f.; siehe hierzu BSK-StGB Heer/Habermeyer (Fn. 22), N 21 zu Art. 59 StGB; Chris Lehner, Freiheitsentziehende Massnahmen im schweizerischen Strafrecht, in: recht 2017, S. 81 ff., S. 88; Godenzi (Fn. 3), S. 14.

52 Nur dieser, nicht aber die akzentuierte dissoziale Persönlichkeit, wies gemäss dem Gutachter eine grosse Bedeutung für das Tatverhalten auf, Urteil des Bundesgerichts 6B_933/2018 vom 3. Oktober 2019 E. 3.5.7.

53 Siehe Urteil des Bundesgerichts 6B_933/2018 vom 3. Oktober 2019 E. 3.5.7.

54 Siehe Urteil des Bundesgerichts 6B_933/2018 vom 3. Oktober 2019 E. 3.5.7.

\section{Verzicht auf die Anwendung international anerkannter Klassifikationssysteme}

29 Die Ausführungen des Bundesgerichts gipfeln wie dargelegt in der Feststellung, eine psychische Störung im Sinne von Art. 59 und 63 StGB setze nicht zwingend eine Diagnose nach den gängigen Klassifikationssystemen (ICD oder DSM) voraus.55 Worauf die Strafjustiz damit verzichtet, beschreibt das Bundesgericht an ebenjener Stelle: Die Manuale sollen «mittels einheitlicher Nomenklatur und Definitionen [...] Transparenz herstellen, Vergleichbarkeit und Nachvollziehbarkeit ermöglichen und so die Zuverlässigkeit (Reliabilität) von Diagnosen verbessern, dies im Hinblick auf die Therapieforschung, die wissenschaftliche Kommunikation über die definierten Störungsbilder und die psychiatrische resp. psychotherapeutische Behandlung».56 Gerade im delikaten Bereich der freiheitsentziehenden Behandlungsmassnahmen, in welchem mehrjährige Therapien (oftmals im geschlossenen Rahmen) regelmässig auch gegen den Willen der Betroffenen durchgeführt werden, darf auf diese Errungenschaften keinesfalls verzichtet werden. Ein Bezug auf ausserhalb des Strafrechts angewandte Massstäbe ist hier besonders wichtig. Freilich bestehen etwa mit dem Risk-Need-ResponsivityModell (RNR-Modell) auch wissenschaftlich fundierte und empirisch validierte

Urteile des Bundesgerichts 6B_933/2018 vom 3. Oktober 2019 E. 3.5.5 und 6B_828/2019 vom 5. November 2019 E. 1.4.

56 Urteil des Bundesgerichts 6B_933/2018 vom 3. Oktober 2019 E. 3.5.5 mit Verweis auf die dort aufgeführten Belegstellen; siehe hierzu namentlich Elmar Habermeyer/Andreas Mokros/Andreas Hill/ Steffen Lau/Henning Hachtel/Marc Graf, Möglichkeiten und Grenzen der Forensischen Psychiatrie, in: forumpoenale 2019, S. 290 ff., S. $291 \mathrm{f}$. 
Interventionsmodelle, die sich nicht zwingend an einer Diagnose nach den gängigen Klassifikationssystemen orientieren.57 Auf diesen Modellen aufbauende unspezifische Interventionen, etwa mittels Lernprogrammen 58 , zeitigen durchaus Erfolge bei der Rückfallprävention. 59 Entsprechende Methoden verstehen sich indes weder als diagnostische Instrumente noch als Alternativen für eine Psychotherapie.60 Anders verhält es sich mit dem den zwei Urteilen des Bundesgerichts zugrunde liegenden FOTRES-Modell. Dieses definiert risikorelevante Aspekte (wie beispielsweise wiederholte Vergewaltigungen) als Diagnose («Vergewaltigungsdispositon»). In der internationalen Fachwelt breit abgestützte Diagnosekriterien werden auf diese Weise durch eigene Risikomerkmale ersetzt, ohne dass diese wissenschaftlich fundiert wären. ${ }^{61}$ Diese Bezugnahme auf je nach Anwenderkreis unterschiedliche Begriffe, die man mit der Etablierung international anerkannter Diagnosemanuale überwunden glaubte, 62 verhindert ein einheitliches und über einzelne Fachgruppen hinaus nachvollziehbares Vorgehen bei der Diagnosestellung. Nur eine verlässlich gestellte Diagnose ermöglicht eine auf die konkrete Störung bezogene

57 Elmar Habermeyer/Andreas Mokros/Peer Briken, «Die Relevanz eines kohärenten forensischen Beurteilungs- und Behandlungsprozesses»: grosser Wurf oder alter Wein in undichtem Schlauch?», in: Forensische Psychiatrie, Psychologie, Kriminologie 2020, S. 4.

58 Siehe hierzu Joder Regli, Lernprogramme, eine Intervention mit viel Potential für den Justizvollzug, in: SZK 2019, S. 7 ff.

59 Jacqueline Bächli-Biétry, Lernprogramme als neue Interventionsform in der Strafjustiz, 2006, Joder Regli, Lernprogramme, eine wirksame Intervention zur Rückfallprävention, in: Strassenverkehr 2019, S. $63 \mathrm{ff}$.

60 Regli (Fn. 58), S. 7.

61 Habermeyer/Mokros/Briken (Fn. 57), S. 5.

62 Habermeyer/Mokros/Briken (Fn. 57), S. 5.
Therapieforschung und damit aussagekräftige Angaben über die Wirksamkeit der Behandlung. ${ }^{63}$ Was das FOTRESManual betrifft, ist jedoch nicht einmal untersucht, geschweige denn belegt, dass dieses Instrument dazu in der Lage ist, mit seinen Merkmalen rückfallrelevante Risiken zu erfassen oder dass die Anwendung von FOTRES sogar dazu befähigen kann, einen Therapieerfolg zu erzielen.64 Zur Effektivität dieser Therapieansätze äussert sich denn auch das Bundesgericht in den zwei dargestellten Urteilen nicht. Ob es die fehlende diesbezügliche Datenlage dabei übersehen oder ignoriert hat, ist nicht entscheidend. Jedenfalls steht fest, dass es die Schleuse für solche (unter Umständen mit langjährigem Freiheitsentzug verbundenen) Behandlungen geöffnet hat, ohne dass sich wissenschaftlich untermauern liesse, dass diese Vorgehensweisen überhaupt in der begutachtenden Disziplin (d.h. der forensischen Psychiatrie) fachlich anerkannt bzw. gar erfolgversprechend anwendbar sind.

\section{Beurteilung des Schweregrads der Störung}

3 Will man auf eine verlässlich festgestellte psychiatrische Diagnose verzichten, wäre es umso wichtiger, die zur Anordnung einer Behandlungsmassnahme berechtigenden psychischen Zustände anhand ihrer Ausprägung einzuschränken. Die forensische Relevanz der «persönlich-

63 Elmar Habermeyer/Steffen Lau/Henning Hachtel/Marc Graf, Der Begriff der psychischen Störung - eine alternativlose Höhenmarke, in: Marianne Heer/Elmar Habermeyer/Stephan Bernard (Hrsg.), Die schwere psychische Störung als Voraussetzung von therapeutischen Massnahmen, 2019, S. 43 ff., S. 47 f.

64 Habermeyer/Mokros/Briken (Fn. 57), S. 4; Habermeyer et al. (Fn. 56), S. 290 ff., S. $291 \mathrm{f}$. 
keitsnahen Risikofaktoren» entscheidet sich gemäss Bundesgericht indes allein danach, wie stark sie in den Anlasstaten zum Ausdruck kommen. Je stärker anders gesagt der Zusammenhang zwischen diesen Risikofaktoren und der zu beurteilenden Delinquenz ist, desto schwerer wiegt nach bundesgerichtlicher Auffassung offenbar die psychische Störung im Rechtssinn. Eine bedeutende Einschränkung des Kriteriums der psychischen Störung ist dadurch nicht zu erreichen. Dass die seelischen Defizite des Täters in der Tat zum Ausdruck kommen müssen, ja der Tat geradezu Symptomcharakter für dessen Störung zukommen muss, ist seit jeher eigenständige Anordnungsvoraussetzung der kriminalrechtlichen Behandlungsmassnahmen.65 Es liegt jedoch auf der Hand, dass auch geringfügigen psychischen Beeinträchtigungen für die Begehung einer Tat überwiegende Bedeutung zukommen können und auf der anderen Seite unbestritten als schwerwiegende psychische Störungen anerkannte Krankheiten bisweilen nur geringen Einfluss auf das Tatgeschehen haben. So kann für einen Straftäter, der an einer Nikotinabhängigkeit leidet, diese Störung von entscheidender Bedeutung für den Diebstahl einer Stange Zigaretten sein. Gleichwohl würde niemand so weit gehen, seine Nikotinabhängigkeit als schwerwiegende psychische Störung hinsichtlich der Anordnung einer strafrechtlichen therapeutischen Massnahme zu bezeichnen. Auf der anderen Seite ist es möglich, dass eine an einer Schizophrenie erkrankte Person dasselbe Delikt begeht, ohne dass diese unbestritten als

65 Siehe Peter Albrecht, Die allgemeinen Voraussetzungen zur Anordnung freiheitsentziehender Massnahmen gegenüber erwachsenen Delinquenten, 1981, S. 48 f.; ferner Stratenwerth (Fn. 23), $\S 8 \mathrm{~N} 20$. schwer geltende Störung einen Einfluss auf das Vermögensdelikt hatte.

\section{Tragweite der neuen}

\section{bundesgerichtlichen Praxis}

31 Zusammenfassend muss konstatiert werden, dass sich das Bundesgericht ohne Not von bewährten medizinischen Konzepten der Diagnosestellung abgewandt hat. Das Anliegen, vorrangig bei Tätern eine therapeutische Massnahme anzuordnen, bei denen die Rückfallwahrscheinlichkeit im Bereich der schwerwiegenden Delinquenz besonders hoch ist, ist - unter den weiteren gegebenen Anordnungsvoraussetzungen - ohne Weiteres zu unterstützen. Mit dem Verzicht auf international anerkannte Diagnosestandards und damit auf die Evaluation der Wirksamkeit von Behandlungen verliert man jedoch das Ziel einer möglichst effektiven Therapie aus den Augen. Es sei erneut darauf hingewiesen, dass in Bezug auf die Merkmale «Dominanzfokus» und «Vergewaltigungsdisposition» keinerlei Therapiestudien vorliegen. Der vom Bundesgericht aufgezeigte Weg ist nicht nur aus medizinisch-ethischer Sicht fragwürdig, sondern stellt auch die Rechtfertigung therapeutischer Massnahmen auf wacklige Beine (nachfolgend lit. a). Ausserdem ergeben sich Abgrenzungsschwierigkeiten zur Verwahrung (nachfolgend lit. b).

\section{a) Legitimation strafrechtlicher Behandlungsmassnahmen}

32 Gerade weil hier primär die Sozialgefährlichkeit des Straftäters und nicht das in der Tat zum Ausdruck gekommene Verschulden Grundlage für die Bemessung des Freiheitsentzugs bildet, bedarf die Sanktionsform der therapeutischen 
Massnahmen einer besonderen Rechtfertigung. Es darf nicht vergessen werden, dass die massnahmeunterworfene Person ein Sonderopfer66 für die Allgemeinheit erbringt, insoweit ihr über die schuldangemessene Strafe hinaus präventiv die Freiheit entzogen wird, das heisst für ein Delikt, das sie nicht begangen hat, sondern nur mit einer gewissen Wahrscheinlichkeit begehen wird. Dass sich die Strafbehörden dabei auf Rückfallprognosen stützen müssen, die nicht durchwegs zu zuverlässigen Ergebnissen führen, 67 verschärft das Problem weiter. Die therapeutischen Massnahmen, die früher mehr als heute auch im wohlverstandenen Interesse der betroffenen Person verstanden wurden, ${ }^{68}$ liessen sich bisher jedenfalls zum Teil auch dadurch legitimieren, dass sie der betroffenen Person halfen, mit ihren Defiziten, die sich auch ausserhalb der Delinquenz zeigten, besser umzugehen.69 Gerade deshalb sind therapeutische Massnahmen in weit grösserem Rahmen zulässig als die rein sichernde Verwahrung. Stützt

66 Siehe treffend Urteil des deutschen Bundesverfassungsgerichts 128, 326 vom 4. Mai 2011 N 101 für die Sicherungsverwahrung nach deutschem Recht.

67 Mario Gmür, Die Gefährlichkeitsprognose, in: AJP 2004, S. 1307 ff., S. 1317 f.; Marianne Heer, in: Marcel Niggli/Hans Wiprächtiger (Hrsg.), Basler Kommentar, Strafrecht I, Art. 1-110 StGB, Jugendstrafgesetz, 4. Aufl. 2019, N 60 ff. zu Art. 64 StGB; Stratenwerth (Fn. 22), 130 f.; Jonas Weber, Zur Verhältnismässigkeit der Sicherungsverwahrung, in: ZStrR 2002, S. 398 ff., S. 400 f.

68 Günter Stratenwerth, strafrechtliche Massnahmen an geistig Abnormen, in: ZStrR 1973, S. 131 ff., S. 135; ders. (Fn. 22), N 29 f. zu § 8; Marianne Heer, in: Marcel Niggli/Hans Wiprächtiger (Hrsg.), Basler Kommentar, Strafrecht I, Art. 1-110 StGB, Jugendstrafgesetz, 4. Aufl. 2019, $\mathrm{N} 7 \mathrm{zu}$ Vor Art. 56 StGB.

69 Günter Stratenwerth, Zur Rechtsstaatlichkeit der freiheitsentziehenden Massnahmen im Strafrecht, in: ZStrR 1966, S. 522; siehe auch Godenzi (Fn. 3), S. 20 und BGE 142 IV 105 E. 5.8.1, BGE 137 IV 201 E. 1.3. man sich bei der Behandlung von Straftätern jedoch nicht mehr primär auf den bewährten allgemeinen medizinischen Erfahrungshintergrund, sondern therapiert rein risikoorientiert, entfällt dieser Aspekt der Rechtfertigung.

33 Will man mit dem Bundesgericht auch die Behandlung (medizinisch kaum fassbarer) Persönlichkeitsmerkmale unter Art. 59 und 63 StGB fassen, ist der Prüfung der Eignung solcher Massnahmen vermehrte Aufmerksamkeit zu schenken. Die Eignung ist eine aus dem Grundsatz der Verhältnismässigkeit fliessende Bedingung zur Rechtmässigkeit jeglicher staatlicher Grundrechtseingriffe.70 Art. 59 und 63 StGB formulieren sie für die therapeutischen Massnahmen entsprechend ausdrücklich als weitere Anordnungsvoraussetzung: Stationäre oder ambulante Therapien dürfen nur angeordnet werden, wenn «zu erwarten ist, dadurch lasse sich der Gefahr weiterer mit dem Zustand des Täters in Zusammenhang stehender Taten begegnen.» ${ }^{71}$ Spätestens an diesem Kriterium dürften die meisten auf einer reinen Risikobeurteilung gemäss FOTRES basierenden Behandlungen nach dem Gesagten scheitern. Niemand kann ernsthaft behaupten, durch eine entsprechende Intervention lasse sich die Legalprognose eines Straftäters erheblich verbessern, wenn keine breit angelegten wissenschaftlichen Studien vorhanden sind, die eine solche Vermutung auch nur andeuten.

34 Schliesslich stellt sich die Frage, welchen bedeutsamen rechtlichen Schranken the-

70 Statt vieler: Giovanni Biaggini, BV Kommentar, Bundesverfassung der Schweizerischen Eidgenossenschaft, 2. Aufl. 2017, N 23 zu Art. 36 BV.

71 Art. 59 Abs. 1 lit. b und Art. 63 Abs. 1 lit. b StGB. 
rapeutische Massnahmen nach der Konzeption des Bundesgerichts überhaupt noch unterliegen. Im zweispurigen Sanktionensystem soll ihnen Ausnahmecharakter zukommen; als primäre Sanktion gilt die schuldangemessene Strafe.72 Indes werden sich kaum mehr Straftäter im Bereich von schwerwiegenden Delikten gegen Leib und Leben, Freiheit und die sexuelle Integrität finden, für die nach der neuen bundesgerichtlichen Praxis eine Massnahme nach Art. 63 oder 59 StGB nicht mehr begründbar ist. Bei rechtswidrig und schuldhaft begangenen Taten lassen sich stets in der Persönlichkeit des Täters liegende und nicht rein situative Gründe dafür ausmachen, wieso er eine bestimmte Tat begangen hat, ob man diese nun als besondere Gewaltbereitschaft, «Dominanzfokus» oder «Vergewaltigungsdisposition» bezeichnet. Dass sich beim Straftäter im zweiten dargestellten Fall des Bundesgerichts das entscheidende, eine Behandlungsmassnahme begründende Merkmal - eine «Vergewaltigungsdisposition» - finden liess, überrascht entsprechend wenig: Ist eine (vorsätzlich und schuldhaft begangene) Vergewaltigung denkbar, ohne Vergewaltigungsfantasien, oder wie sie der Gutachter umschrieb,73 ohne den «Wunsch nach sexuellen Handlungen gegen den Willen der betroffenen Person»?

35 Es geht hier keinesfalls darum, die zwei abgeurteilten Taten zu bagatellisieren. Der Schuldbefund wie auch das hohe

72 Bommer (Fn. 21), S. 28; Marianne Heer, in: Marcel Niggli/Hans Wiprächtiger (Hrsg.), Basler Kommentar, Strafrecht I, Art. 1-110 StGB, Jugendstrafgesetz, 4. Aufl. 2019, N 35 zu Art. 56 StGB.

73 Urteil des Bundesgerichts 6B_828/2019 vom 5. November 2019 E. 1.4.
Strafmass waren denn auch in den bundesgerichtlichen Verfahren nur noch Randthemen. Die therapeutischen Massnahmen sollen aber gerade nicht jeden besonders verwerflich vorgehenden Straftäter per se erfassen, sondern nur jenen, der an einem besonders ausgeprägten psychischen Defizit leidet und mit guten Chancen behandelt werden kann. Dass dabei die Minimierung des Rückfallrisikos Massstab wie auch Ziel ist, ist richtig. Die Auswahl der dazu geeigneten Kandidaten soll jedoch - anders als bei der rein sichernden Verwahrung danach geschehen, wie hoch die reelle Chance der Behandlung ist.

\section{b) Verhältnis der neu konzipierten Behandlungsmassnahmen zur Verwahrung}

36 Die schweizerische Literatur und Rechtsprechung haben sich ausführlich mit der Frage beschäftigt, wie die stationären therapeutischen Massnahmen zur Verwahrung abzugrenzen sind.74 Dies aus gutem Grund: Die rein sichernde, erst im Nachgang zur Verbüssung einer Freiheitsstrafe vollzogene Verwahrung untersteht aus den bereits genannten Gründen75 deutlich einschneidenderen Voraussetzungen als ambulante und stationäre Therapien. Sie soll - bei fehlender Behandelbarkeit - nur als ultima ratio angeordnet werden. ${ }^{76}$ Die nun anstehende Pathologisierung zahlreicher Straftäter führt zum einen dazu, dass neu auch Delinquenten mittels strafrechtlicher Behandlungsmassnahmen die Freiheit entzogen werden kann, für die solche Mass-

$74 \overline{\text { Hierzu Chris Lehner, Nachträgliche Anordnung }}$ stationärer therapeutischer Massnahmen, 2015, Rz. 14.

75 Siehe vorne, Rz 32.

76 BGE 134 IV 315 E. 3.4.2 und 3.5. 
nahmen mangels schwerer psychischer Störung bisher nicht möglich waren und bei denen die Anordnung der Verwahrung, etwa wegen des Fehlens einer qualifizierten Anlasstat, nicht in Betracht kam.77 Auf der anderen Seite müsste nicht nur für zahlreiche, sich heute in der Verwahrung befindliche Verurteilte in Anwendung von Art. 65 Abs. 1 StGB nachträglich stattdessen eine stationäre therapeutische Massnahme angeordnet werden. Für viele Delinquenten, denen bisher die Verwahrung drohte, wird ausserdem künftig eine Massnahme nach Art. 59 StGB angeordnet werden müssen, da sie die tiefliegenden Voraussetzungen dieser Massnahme nun erfüllen. Was sich für die Betroffenen zunächst als weniger schwere Sanktion anhören mag, entpuppt sich bei Personen mit negativem Behandlungsverlauf spätestens dann als blossen Aufschub des Problems, wenn die Vollzugsbehörde die therapeutische Massnahme nach jahrelanger erfolgloser Therapie wegen Aussichtslosigkeit aufhebt und in Anwendung von Art. 62c Abs. 4 StGB die Ersetzung durch eine Verwahrung beantragt.

In solchen Fällen wäre es für alle Beteiligten besser, auf das Schüren falscher Behandlungshoffnungen zu verzichten. Bei hoch rückfallgefährdeten Straftätern, die schwerwiegende Delikte begangen haben und nicht behandelbar sind oder bei denen die Behandelbarkeit nicht evaluiert ist, wäre es am ehrlichsten, wenn das Sachgericht keine therapeutische Massnahme anordnete, sondern bereits zu diesem Zeitpunkt entschiede, ob der Straftäter «bloss» seine schuldangemessene Strafe zu verbüssen hat, oder im

77 Illustrativ dazu das bei Godenzi (Fn. 3) S. 3 ff. aufgeführte Beispiel.
Anschluss daran zusätzlich verwahrt werden soll. Das erstinstanzliche Gericht ist im erstkommentierten Fall genau so vorgegangen. Eine viele Jahre dauernde Therapie ohne merklichen Nutzen ist hingegen teuer und unmenschlich, ausserdem fehlen dadurch knappe Behandlungsplätze für tatsächlich therapierbare Straftäter. Ebenso fragwürdig und ethisch nicht vertretbar ist die Behandlung auf Probe - eine Behandlung in Unkenntnis des zu erwartenden Behandlungserfolgs. Es handelt sich dabei letztlich um zwangsweise angeordnete medizinische Experimente, die auf Kosten der Freiheit der betroffenen Person durchgeführt werden.

\section{c) Umgang mit der neuen Praxis}

38 Es ist im Zusammenhang mit den zwei kommentierten Urteilen bereits zu Recht darauf hingewiesen worden, dass es jedem massnahmenanordnenden Gericht obliegt, sich unabhängig davon zu überzeugen, ob im konkreten Fall eine schwere psychische Störung vorliegt, die das Aussprechen einer Behandlungsmassnahme rechtfertigt. ${ }^{8}$ In gleicher Weise hat es sich zu vergewissern, ob das beigezogene forensisch-psychiatrische Gutachten lege artis erstellt wurde und nicht nur im Ergebnis, sondern auch bei der Herleitung der Befunde überzeugt.

39 Nun wird bereits die Mehrheit der in der Schweiz praktizierenden forensischen Psychiaterinnen und Psychiater die Feststellung von - wie auch immer gearteten - Risikomerkmalen (auch im Zusammenwirken) nicht als psychische Störung bezeichnen, sofern sich ein solcher Befund nicht auf ein Klassifikationssystem

78 Bommer (Fn. 21), S. 31. 
wie ICD oder DSM stützen kann. Noch weniger wird eine solche Sachverständige bzw. ein solcher Sachverständiger diese Merkmale ohne reliable Diagnose als nach medizinischen Kriterien schwerwiegend bezeichnen. Auch die im erstkommentierten Fall zunächst beigezogene Gutachterin hatte darauf hingewiesen, dass die gesicherte Diagnose einer psychischen Störung, namentlich einer narzisstischen Persönlichkeitsstörung, im konkreten Fall nicht möglich sei.79 Mit Blick auf die psychiatrische Fachliteratur lässt sich denn auch in Zukunft von gerichtlicher Seite weiterhin argumentieren, die von der sachverständigen Person festgestellten Risikomerkmale entsprächen nicht einer nach den Regeln der ärztlichen Kunst gestellten Diagnose und liessen den Schluss auf eine psychische Störung im rechtlichen Sinne nicht zu. Spätestens wird die Anordnung einer therapeutischen Massnahme in vielen vergleichbaren Fällen schliesslich an der Voraussetzung des zu erwartenden Behandlungserfolgs respektive der Eignung der Massnahme scheitern.

Zudem bleibt abschliessend zu hoffen, dass sich einige kantonale Gerichte trotz den zwei entgegenstehenden Urteilen des Bundesgerichts an die unmissverständlichen Vorgaben des Gesetzes halten, und auch künftig für die Anordnung einer therapeutischen Massnahme die Diagnose einer schweren psychischen Störung im medizinischen Sinne voraussetzen. Art. 191c BV und Art. 4 Abs. 1 StPO halten denn die Gerichte (auch bei abweichender höchstrichterlicher Praxis) zu einer unabhängigen Prüfung der Rechtslage an. Ohne gesetzliche Grundlage eine (unter Umständen mehrjährige freiheitsentziehende) Behandlungsmassnahme anzuordnen, dürfte nicht weniger heikel sein, als von der Rechtsprechung des Bundesgerichts in diesem Punkt abzuweichen. das zum oben dargestellten Schluss gelangte. 